\title{
El factor distancia en la atracción de los centros comerciales. Una aplicación a la ciudad de Aracaju (Sergipe, Brasil)
}

\author{
José Edgard Da Mota Freitas (efreitas@infonet.com.br) \\ Ana Isabel Rodríguez Escudero (ana@eco.uva.es) \\ Departamento de Economía y Administración de Empresas \\ Facultad de Ciencias Económicas y Empreariales \\ Avda. Valle Esgueva, $n^{0} 647011$ Valladolid
}

\begin{abstract}
RESUMEN
La elección de un centro comercial por un individuo es el resultado de comparar la utilidad de las diversas alternativas. La literatura previa nos lleva a proponer una función de utilidad del consumidor integrada por dos componentes: el factor espacial y el factor imagen. El factor espacial refleja la influencia que sobre el comportamiento del individuo tiene la posición en el espacio geográfico de una determinada alternativa de compra, esto es: el coste -en tiempo, dinero y esfuerzoque supone acceder al centro. Si el consumidor tuviera que elegir entre dos centros iguales en un mercado perfectamente uniforme escogería el que estuviera más próximo, sin embargo, puesto que lo más probable es que existan ciertos atributos diferenciales que doten a un centro de mayor atractivo que a otro ante una determinada decisión de compra, cabe pensar que cuando la diferencia en la distancia entre los centros no supere un determinado nivel o umbral, se convierta en un criterio irrelevante de elección. Es decir, ante dos centros diferentes, el consumidor puede estar dispuesto a soportar cierto coste de desplazamiento adicional si ello le permite acceder a mejores ofertas. La verificación de estos supuestos constituye el objetivo de este trabajo. Para ello contamos con la información obtenida de una encuesta personal con cuestionario estructurado, dirigido a 350 individuos que participan activamente en las decisiones de compra familiares en los hogares de Aracaju (Brasil).
\end{abstract}

\section{SUMMARY}

The election of a mall for an individual is the result of comparing the utility of the diverse alternatives. The previous literature leads us to propose a function of utility of the consumer integrated by two components: the spatial factor and the factor image. The spatial factor reflects the influence that on the behavior of the individual has the position in the geographical space of a certain alternative of buy, this is: the cost in time, money and effort that supposes acceding to the center. If the consumer had to choose among two equal centers on a perfectly uniform market, he would choose the one that was more close, nevertheless, since the most probable thing is that exist certain differential attributes that provide to a center of major attraction that to other one, it is necessary to think that, when the difference in the distance among the centers does not overcome a certain level or threshold, is converted into an irrelevant criterion of election. That is to say, before two different centers, the consumer can be prepared to support certain cost of additional displacement if it him allows to accede to better offers. The monitoring of these suppositions constitutes the aim(lens) of this work. For it we rely on the information obtained of a personal survey questionnaire constructed, directed 350 individuals who take part in the familiar decisions of buy in Aracaju's homes (Brazil).

\section{Concepto y características del centro comercial}

Frente a las particularidades del comercio independiente e integrado, el comercio asociado se caracteriza por la unión de varios intermediarios con el objeto de lograr un mayor poder dentro del canal, pero manteniendo la propiedad jurídica de sus establecimientos. Podemos distinguir dos tipos de asociacionismo comercial: asociacionismo de base contractual y asociacionismo espacial (véase el cuadro 1). Estamos ante una forma de asociacionismo contractual cuando una serie de empresas independientes de distintos niveles de producción y distribución integran sus programas de marketing mediante contrato, con objeto de obtener ciertas economías. Por este procedimiento se consigue la ampliación de las redes de distribución sin tener que realizar grandes inversiones; es el caso de las centrales de compras, las cadenas voluntarias o las franquicias. El asociacionismo espacial, otra forma de coordinar las acciones comerciales manteniendo la independencia de gestión, responde a la concentración en un mismo espacio geográfico de varios intermediarios; en este tipo de asociación se clasifica el centro comercial y la zona comercial. 
Revista Eletrônica de Ciência Administrativa (RECADM) - ISSN 1677-7387 Faculdade Cenecista de Campo Largo - Coordenação do Curso de Administração v. 2, n. 1, maio/2003 - http://revistas.facecla.com.br/index.php/recadm/

CUADRO 1

Tipología del comercio

\begin{tabular}{|c|c|c|}
\hline COMERCIO INDEPENDIENTE & COMERCIO INTEGRADO & COMERCIO ASOCIADO \\
\hline $\begin{array}{ll}\text { B } & \text { Mayoristas independientes } \\
\text { B } & \text { Minoristas independientes }\end{array}$ & $\begin{array}{ll}\text { B Cooperativas de consumo } \\
\text { ß } & \text { Cadenas sucursalistas (grandes } \\
& \text { almacenes, hipermercados, } \\
\text { supermercados, tiendas de } \\
\text { descuento...) }\end{array}$ & $\begin{array}{ll}\text { B } & \begin{array}{l}\text { Asociacionismo contractual } \\
\text { (cadenas voluntarias, centrales de }\end{array} \\
\text { compras, franquicias...) } \\
\text { B } \\
\text { Asociacionismo espacial (centros } \\
\text { comerciales). }\end{array}$ \\
\hline
\end{tabular}

Como forma de agrupación horizontal en la que existe concentración espacial, el origen del centro comercial hay que situarlo en torno a la década de los 50 del siglo pasado, momento que coincide con la expansión urbana hacia la periferia gracias a la creciente mejora de los sistemas de transporte. Consecuentemente, surge la necesidad de establecer un comercio que atienda a los barrios distantes del centro de la ciudad (Manzo y Kunto, 1975). Cuando las personas empezaron a moverse hacia la periferia de las ciudades, el centro de la ciudad, con sus problemas de saturación, comenzó a perder una cuota de sus negocios, por lo que los comerciantes abrieron sucursales en otras localizaciones.

Vinculadas con el aumento de la renta, la población y el parque automovilístico, las décadas de los 60 y 70 son la edad de oro en la expansión de los centros comerciales. Su crecimiento se ve potenciado por la aparición, a partir de 1975, de los hipermercados, que se erigen en la "locomotora" fundamental de los centros comerciales europeos. Los años 80 se caracterizan por un acusado aumento de las actividades de ocio como factor de atracción.

Un centro comercial (o shopping centre) es un conjunto de negocios detallistas independientes, planificados, desarrollados y dirigidos como una unidad, que se presenta ante su entorno con una imagen única. Entre los negocios o establecimientos cabe encontrar grandes almacenes, hipermercados, supermercados, tiendas especializadas, servicios, etc. Uno de estos establecimientos ejercerá de "locomotora", "magneto" o "ancla", es decir, de tienda que atraerá a los consumidores hacia el núcleo comercial. Para un shopping localizado en el centro de una ciudad, la tienda "locomotora" generalmente es un gran almacén; para un shopping de periferia es casi siempre un hipermercado.

Las principales características que cabe atribuir a un centro comercial son las siguientes: la unidad de ubicación en una o varias edificaciones, la unidad de gestión, la existencia de un nombre característico, la utilización de servicios y estructuras comunes (aparcamiento, vigilancia, sanidad...) y la gestión independiente y diversificada de la oferta comercial.

Una cuestión central para el éxito y progreso de un centro comercial es la elección de un adecuado mix de arrendatarios o combinación de establecimientos comerciales. Las tiendas son alquiladas por los comerciantes al administrador del centro, al que pagan un alquiler. Tradicionalmente los grandes almacenes, los supermercados y las tiendas especializadas han dominado el mix de los centros comerciales (Carlson, 1991). No obstante, con la aparición de los llamados "down-sized" y los shoppings temáticos, otros tipos de establecimientos (restaurantes, bancos, peluquerías, etc.) están asumiendo un papel prominente. De acuerdo con Kayling (1973), un mix óptimo debe caracterizarse por una diversificación equilibrada de tiendas que aporte la variedad necesaria para atraer a la población al centro, un potencial máximo de ventas en el área, la existencia de sinergias entre los arrendatarios satélites, un ambiente de compras agradable y un retorno sobre la inversión adecuado.

\section{La atracción comercial}

De acuerdo con las tradicionales etapas del proceso de decisión (reconocimiento de las necesidades, búsqueda de información, evaluación de alternativas y decisión), extensamente desarrollado en la literatura del marketing, el proceso de elección de un centro por un individuo es resultado de comparar la utilidad o beneficio esperado de las diversas alternativas. La atracción comercial, por tanto, está relacionada con todos aquellos factores que guían al consumidor hacia un determinado núcleo comercial porque permiten maximizar la relación utilidad / coste. Las conclusiones de estudios previos nos llevan a proponer una función de utilidad integrada por dos componentes: el factor espacial y el factor imagen. Veamos, a continuación, cuáles son las formas principales de medir estos factores, así como las variables que se utilizan para medir la atracción comercial. 
Revista Eletrônica de Ciência Administrativa (RECADM) - ISSN 1677-7387

Faculdade Cenecista de Campo Largo - Coordenação do Curso de Administração

v. 2, n. 1, maio/2003 - http://revistas.facecla.com.br/index.php/recadm/

\subsection{Medición del factor espacial}

El factor espacial refleja la influencia que sobre el comportamiento del individuo tiene la posición en el espacio geográfico de una determinada alternativa de compra. Representa el coste -en tiempo, dinero y esfuerzo- que supone acceder al centro. Los cuatro acercamientos o enfoques alternativos para la medición de esta variable han sido (Suárez Vázquez, 1999):

B La distancia objetiva que separa a los individuos de los diferentes centros (Hauser y Koppelman, 1979; Wee y Pearce, 1985; Finn, McQuitty y Rigby, 1994: Rust y Donthu, 1995; Borgers y Timmermans, 1987), como, por ejemplo, el número de kilómetros. Esta forma de medición no tiene en cuenta aspectos tales como los atascos o los semáforos, que pueden dar lugar a que distancias reales muy cortas se tarden mucho tiempo en recorrer.

B El tiempo de desplazamiento objetivo, enfoque que tiene en cuenta la existencia de ciertos costes de desplazamiento que no están en relación con la distancia física, ya que en ocasiones el esfuerzo requerido para acceder a un centro no está necesariamente correlacionado con la distancia. El indicador más utilizado es el tiempo de desplazamiento. Esta alternativa tiene la limitación de que normalmente se trata de datos basados en el desplazamiento medio de un vehículo tipo desde la zona de origen a la zona de destino (Gautschi, 1981; Weisbrod, Parcells y Kern, 1984).

ß Tiempo de desplazamiento subjetivo (estimado por el comprador), forma de medición del factor espacial que pone de relieve su concepción como una medida del coste de oportunidad que tiene destinar tiempo a esta actividad concreta de compra en lugar de a opciones alternativas. El tiempo de desplazamiento subjetivo se ha venido midiendo de dos formas distintas:

B Por medio de una escala de razón, solicitando al comprador que aproxime el tiempo medio que tarda en desplazarse a cada centro (Nevin y Houston, 1980; Stolman, Gentry y Anglin, 1991; McGoldrick, 1992; Más, 1996a y b y 1999).

B A través de una escala de intervalo en la que el consumidor valora la proximidad al establecimiento analizado, lo que da entrada al esfuerzo del consumidor no medido por el propio coste temporal (Howell y Rogers, 1980; Arnold, Roth y Tigert, 1981).

\subsection{Medición del factor imagen}

La literatura sobre atracción comercial generalmente asume que la imagen percibida del centro comercial -0 modo en que el centro se define en la mente del comprador-, explica, junto con la ubicación geográfica, la elección y preferencia por un centro comercial.

Aun cuando han proliferado los estudios sobre la imagen de establecimientos individuales, y los resultados se han tratado de extrapolar a los centros comerciales, la idoneidad de esta traslación se ve cuestionada por dos aspectos: 1) algunos atributos de imagen, como la relación calidad-precio o la atención personal, sólo tiene sentido aplicarlos si el centro ha conseguido proyectar una imagen común; y, 2) el estudio de los centros comerciales requiere contemplar atributos que no tienen cabida al analizar la imagen de los puntos de venta aislados (Suárez Vázquez, 1999). Es importante, pues, antes de valorar la imagen de un centro, probar con todas aquellas variables que puedan determinarla para comprender mejor sus dimensiones. En el cuadro 2 se muestran los resultados de los análisis factoriales efectuados a los indicadores de partida para un conjunto de ocho trabajos. 
Revista Eletrônica de Ciência Administrativa (RECADM) - ISSN 1677-7387

Faculdade Cenecista de Campo Largo - Coordenação do Curso de Administração

v. 2, n. 1, maio/2003 - http://revistas.facecla.com.br/index.php/recadm/

CUADRO 2

Factores representativos de la variable imagen

\begin{tabular}{|c||l||}
\hline \multicolumn{1}{|c||}{ Estudio } & \multicolumn{1}{|c|}{ Factores derivados de los indicadores individuales de imagen } \\
\hline \hline Nevin y Houston (1980) & Variedad de beneficios ofrecidos por el área, Equipamiento, Posicionamiento. \\
\hline \hline Más (1996b) & Ambiente de compra desenfadado, Calidad del servicio prestado, Posicionamiento. \\
\hline \hline Wee (1986) & Variedad, Equipamiento, Mantenimiento, Operatividad. \\
\hline \hline Hauser y Koppelman (1979) & Variedad, Calidad y satisfacción, Valor, Aparcamiento. \\
\hline \hline McGoldrick (1992) & $\begin{array}{l}\text { Experiencia de ocio, Servicio al cliente, Establecimiento, Calidad del ambiente, } \\
\text { Facilidad para utilizar el coche, Aglomeración, Facilidad para utilizar el autobús, } \\
\text { Precios. }\end{array}$ \\
\hline \hline Howell y Rogers (1980) & Ambiente, Personal, Moda, Publicidad, Accesibilidad. \\
\hline \hline Stolman et al .(1991) & $\begin{array}{l}\text { Surtido, Ocio, Ambiente, Precios, Variedad, Ventas, Equipamiento, Conocimiento, } \\
\text { Relaciones sociales. }\end{array}$ \\
\hline \hline Gentry y Burns (1977-78) & Oferta, Aparcamiento, Establecimiento, Horario. \\
\hline \hline
\end{tabular}

FUENTE: De Juan y Rivera (1998).

\subsection{Medición de la atracción comercial}

A la hora de medir esta variable, también existe cierta disparidad en las propuestas (véase el cuadro 3). De los resultados derivados de las distintas aproximaciones empíricas, la frecuencia relativa de viajes en el último año resulta ser una de las medidas más utilizadas, tanto de forma individual como combinada con otras variables. Aquellos autores que utilizan esta misma variable contemplando un periodo de tiempo más extenso (tres años) o más restringido (última semana), sobrevaloran o infravaloran el comportamiento del consumidor, ya que es difícil que este recuerde exactamente todos su viajes de compra durante los últimos años o que su comportamiento en la semana anterior represente el comportamiento a lo largo del tiempo. El gasto efectuado y la frecuencia de compras son las siguientes medidas en importancia, si nos atenemos a las veces que se utilizan.

CUADRO 3

Medición de la atracción comercial

\begin{tabular}{|c|c|}
\hline Estudio & Indicador utilizado \\
\hline Nevin y Houston (1980) & $\begin{array}{ll}\text { B } & \text { Probabilidad de compra. } \\
\text { B } & \text { El comportamiento real de compra. }\end{array}$ \\
\hline Gautschi (1981); Más (1996a) & ß Frecuencia de visitas durante los últimos doce meses. \\
\hline Wee y Pearce (1985); Wee (1986) & $\begin{array}{ll}\text { B } & \text { Frecuencia de visitas. } \\
\text { B } & \text { Gastos realizados en cada visita. }\end{array}$ \\
\hline McGoldrick (1992) & $\begin{array}{ll}\text { ß } & \text { Frecuencia de las vistas } \\
\text { ß } & \text { Duración de las visitas. } \\
\end{array}$ \\
\hline Finn et al. (1994) & ß Frecuencia de las visitas. \\
\hline Howell y Rogers (1994) & $\begin{array}{ll}\text { ß } & \text { Frecuencia de las visitas. } \\
\text { ß } & \text { Gastos realizados. } \\
\text { ß } & \text { Números de semanas desde la última compra. }\end{array}$ \\
\hline Stolman et al. (1991) & $\begin{array}{ll}\text { ß } & \text { Frecuencia de compra. } \\
\text { ß } & \text { Intenciones de compra. } \\
\end{array}$ \\
\hline Weisbrod et al. (1984) & ß Frecuencia de visitas. \\
\hline
\end{tabular}

Quede claro que estas formas de medición de la atracción son indicadores que se refieren a diferentes niveles de respuesta del consumidor. No se trata de conceptos intercambiables, sino que cada uno hace referencia a comportamientos diferentes (Spiggle y Sewall, 1987). 


\subsection{Objetivos de la investigación}

Como ya hemos dicho, uno de los factores clave para determinar la atracción comercial de un centro es la distancia. El postulado, desarrollado en los años 30 y 40, y propuesto por la teoría de los lugares centrales establecía que la elección de un centro comercial en un mercado perfectamente uniforme dependía únicamente de la distancia, lo que llevaba a predecir que el individuo visitaría siempre aquel establecimiento más próximo. Sin embargo, tras la breve exposición de la literatura sobre atracción comercial, considerar que un individuo tiene que concentrar sus compras tan sólo en el centro que le resulte más próximo parece un supuesto poco realista. Esto da lugar a considerar como hipótesis de trabajo la siguiente proposición:

H1. Dentro de una determinada zona espacial, los individuos ni visitan ni compran en un único centro, sino que tienden a visitar y repartir sus compras entre varios de ellos.

Ahora bien, postular que un consumidor muestra intención de visitar y de comprar en todos o casi todos los centros comerciales de una determinada zona, no quiere decir que consideremos irrelevante la distancia en la elección comercial, puesto que la intención de visita y compra -aunque no sea cero para los centros más alejados-, puede decrecer cuando aumenta la distancia, especialmente si estamos antes la elección de centros de idénticas o muy análogas características. El supuesto más realista en este caso será considerar que la probabilidad de elección decrecerá con la distancia.

No obstante, puesto que siempre existirán ciertos atributos diferenciales que doten a un centro de interés frente a otro, y viceversa (bien una tienda concreta, la facilidad de aparcamiento, el horario de cierre...), es probable que el efecto de la distancia se vea distorsionado por estas particularidades, y que el consumidor, si la diferencia en la distancia entre los centros no supera un determinado nivel o umbral, considere este criterio irrelevante frente a otros criterios relacionados con la imagen del centro o con su atractivo para la compra de determinado producto o servicio. Es decir, ante dos centros diferentes, el consumidor puede estar dispuesto a soportar cierto coste adicional de desplazamiento si ello le permite acceder a mejores ofertas. Así las cosas, estimamos que:

H2. Cuando la diferencia en distancia entre los centros comerciales no alcanza un determinado nivel o umbral, ésta variable no se muestra relevante en la atracción de un centro comercial.

No obstante, a pesar de que el desarrollo urbano y el incremento generalizado de la movilidad espacial ha reducido considerablemente la importancia de la conveniencia espacial como determinante de la compra, difícilmente este factor puede convertirse en irrelevante ${ }^{1}$ cuando la distancia entre dos centros es notable. Por ello postulamos que:

H3. Cuando la diferencia en distancia de los centros empieza a ser alta, se manifiesta su relevancia sobre la atracción comercial.

Dada la muestra con la que contamos, nuestro objetivo se centra en la verificación de las dos primeras hipótesis, ya que se trata de una ciudad de tamaño pequeño-mediano, en la que la distancia a los dos centros existentes más o menos amplia- es similar para un determinado consumidor. Concretamente, el tiempo medio de desplazamiento en el transporte habitual está en 15,4 y 20,7 minutos a cada uno de los dos centros que hay en esta ciudad.

Además, como puede verse en el epígrafe posterior relativo a la caracterización de los centros, estos son lo suficientemente diferentes, incluso en su nivel medio de éxito (uno de ellos es bastante más visitado y la duración de las visitas es notablemente mayor), como para plantearnos la irrelevancia de la distancia.

Para el contraste y prueba de las hipótesis formuladas necesitamos medir las variables atracción comercial y distancia. Puesto que, como ya hemos visto, en la revisión de la literatura se plantea el problema empírico de la selección de las variables a utilizar, porque los distintos autores proponen alternativas muy diversas en cuanto a la forma de medición de las variables, hemos considerado varios indicadores, tanto de la variable dependiente la atracción- como de la variable independiente -la distancia- (véase el cuadro 4), a fin de no presentar resultados que puedan estar influidos por la elección de unas variables concretas.

\footnotetext{
${ }^{1}$ Los trabajos recientes de Medina (1998) y González Benito (2000) prueban la relevancia que sigue teniendo la distancia en la elección comercial.
} 


\section{Revista Eletrônica de Ciência Administrativa (RECADM) - ISSN 1677-7387 Faculdade Cenecista de Campo Largo - Coordenação do Curso de Administração v. 2, n. 1, maio/2003 - http://revistas.facecla.com.br/index.php/recadm/}

Particularmente, la medición de la variable distancia se ha realizado tomando medidas de tiempo de desplazamiento subjetivo porque este enfoque ayuda a poner de relieve la concepción del factor espacial como una medida del coste de oportunidad que el consumidor tiene que destinar tiempo a esa actividad concreta de compra en lugar de a opciones alternativas.

\section{CUADRO 4}

Variables utilizadas

\begin{tabular}{|c|c|}
\hline Variables de atracción comercial & Variables de distancia \\
\hline $\begin{array}{l}\text { A1. Frecuencia de visitas. } \\
\text { A2. Duración de las visitas (en minutos). } \\
\text { A3. Gasto mensual. } \\
\text { A4. Intención de visita. }\end{array}$ & $\begin{array}{l}\text { D1. Tiempo de desplazamiento en minutos usando } \\
\text { los medios habituales de transporte. } \\
\text { D2. Proximidad al centro. }\end{array}$ \\
\hline
\end{tabular}

\section{Diseño de la investigación}

En este apartado se expone, en primer lugar, los rasgos más destacados de los shoppings que nos sirven para cumplir con los objetivos del trabajo, en segundo lugar, la forma de recogida de la información, y finalmente, una primera descripción de la muestra.

\subsection{Los shoppings centres en la ciudad de Aracaju}

Con un nivel de calidad similar al de los países desarrollados, Brasil es el décimo país del mundo en cantidad de shoppings operativos. Concretamente, en enero de 2001 funcionaba un número de 156 centros comerciales artificiales, que daban empleo a 285.704 personas. Desde la inauguración del primero, en 1966, el número se ha ido duplicando cada cinco años. (Véase la evolución de las ventas en la figura 1.)

\section{FIGURA 1}

Evolución de los centros comerciales en Brasil

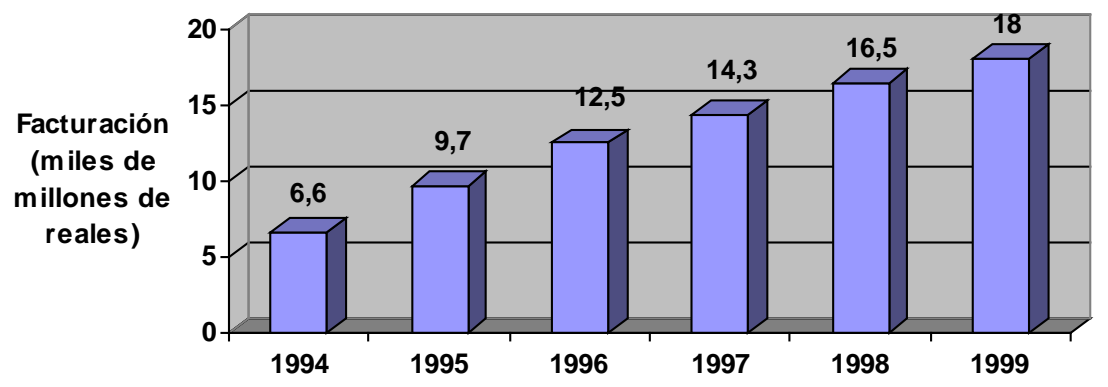

En el estado de Sergipe, concretamente en su capital, Aracaju, hay dos shoppings centres: Rio Mar y Jardins. El primero en crearse fue el Rio Mar, centro comercial que se ubica en una de las zonas más valoradas y con mejores accesos de la ciudad - tanto si se utiliza transporte público como vehículo propio-. El segundo de los shopping, el Jardins, se inauguró en 1999 y desde entonces ya ha pasado por varias ampliaciones. Desde los $9.000 \mathrm{~m}^{2}$ de superficie inicial, ha alcanzado los $20.000 \mathrm{~m}^{2}$. Cuenta con 120 tiendas, entre las que destaca un supermercado, un C\&A y 10 salas de cine. Está ubicado un poco más próximo al centro de la ciudad que el shopping Rio Mar.

Como se puede ver en el Cuadro 5, las principales diferencias entre los shoppings las establecen: el ocio insignificante en el Rio Mar y al que en el Jardins se le dedica una superficie superior al 20\% del total-, los artículos varios -también mucho más importantes en el Jardins-, y la superficie desocupada -casi la mitad de la total en el Rio Mar y despreciable en el Jardins. 
Revista Eletrônica de Ciência Administrativa (RECADM) - ISSN 1677-7387

Faculdade Cenecista de Campo Largo - Coordenação do Curso de Administração

v. 2, n. 1, maio/2003 - http://revistas.facecla.com.br/index.php/recadm/

CUADRO 5

Reparto de la actividad en los shoppings de Aracaju

\begin{tabular}{|c|c|c|c|c|}
\hline & \multicolumn{2}{|c|}{ Rio Mar } & \multicolumn{2}{|c|}{ Jardins } \\
\hline & Área $\left(\mathrm{m}^{2}\right)$ & Porcentaje & Área $\left(\mathrm{m}^{2}\right)$ & Porcentaje \\
\hline Supermercado & 3.463 & 22,4 & 6.200 & 29,0 \\
\hline Vestido y calzado & 2.578 & 16,7 & 4.285 & 20,0 \\
\hline Tiendas conveniencia & 323 & 2,1 & 110 & 0,5 \\
\hline Artículos para el hogar & 204 & 1,3 & 680 & 3,2 \\
\hline Servicios & 606 & 3,9 & 1.324 & 6,2 \\
\hline Alimentación & 419 & 2,7 & 586 & 2,7 \\
\hline Ocio & 118 & 0,8 & 4.670 & 21,8 \\
\hline Artículos varios & 783 & 5,1 & 3.423 & 16,0 \\
\hline Área sin ocupación & 6.936 & 45,0 & 108 & 0,5 \\
\hline TOTAL & 15.430 & 100,00 & 21.386 & 100,00 \\
\hline
\end{tabular}

El conjunto de individuos que se estima que forman parte de la zona de influencia de los dos shoppings es de 467.000 habitantes. El área primaria, que se extiende hasta $5 \mathrm{~km}$. a la redonda, es la que aporta la mayor parte de las ventas (en torno a un 75\%). El área secundaria, que abarca hasta, aproximadamente, los $15 \mathrm{~km}$., reúne a consumidores bastante dispersos que vienen a suponer un $15 \%$ de las ventas. Finalmente, el área terciaria se extiende hasta $25 \mathrm{~km}$. y comprende toda la zona metropolitana. Aunque el potencial de consumo de la región no es alto, se estima que la demanda existente posibilita la convivencia de dos shopping centres en la ciudad de Aracaju; no obstante, no es suficiente para la instalación de un nuevo shopping.

CUADRO 6

Datos demográficos de la ciudad de Aracaju

\begin{tabular}{|c|c|c|}
\hline Área de influencia & Habitantes & Vivienda \\
\hline $\begin{array}{l}\text { Primaria } \\
\text { Secundaria } \\
\text { Terciaria }\end{array}$ & $\begin{array}{c}42.169 \\
317.876 \\
106.761\end{array}$ & $\begin{array}{l}12.477 \\
92.368 \\
25.755 \\
\end{array}$ \\
\hline TOTAL & 466.806 & 130.600 \\
\hline
\end{tabular}

FUENTE: Ayuntamiento de Aracaju

\subsection{La recogida de información}

La información básica fue obtenida de una encuesta personal con cuestionario estructurado, dirigido a los individuos que participan activamente en las decisiones de compra familiares en los hogares de Aracaju. La muestra esta integrada por 350 individuos, la mitad de ellos entrevistados en el shopping Rio Mar y la otra mitad en Jardins. Los encuestados fueron seleccionados de forma aleatoria en la semana del 15 al 22 de mayo de 2002 durante el horario normal de funcionamiento de los dos centros, de 10 a 22 horas (véase la ficha técnica en el Cuadro 7).

CUADRO 7

Ficha técnica

\begin{tabular}{||l||l||}
\hline \hline Universo & Compradores de centros comerciales \\
Ámbito & Ciudad de Aracaju, en el estado de Sergipe (Brasil) \\
Tamaño de la muestra & 350 \\
Error muestra & $5,2 \%$ \\
Fecha del trabajo de campo & Mayo de 2002 \\
\hline
\end{tabular}

Las variables relativas a la evaluación de los atributos de los centros y el nivel de compras y uso de los diferentes productos y servicios, fueron medidas en una escala Likert de 5 puntos, donde 1 significa "muy bajo" y 5 "muy alto". Las variables sociodemográficas consideradas han sido: la edad, el sexo, el estado civil, el nivel de formación, la posesión de vivienda y el nivel de ingresos. 
Revista Eletrônica de Ciência Administrativa (RECADM) - ISSN 1677-7387

Faculdade Cenecista de Campo Largo - Coordenação do Curso de Administração

v. 2, n. 1, maio/2003 - http://revistas.facecla.com.br/index.php/recadm/

\subsection{Descripción de la muestra}

Los dos shoppings centres son igualmente conocidos por la población de Aracaju; de hecho, todos los encuestados afirman haberlos visitado y haber comprado en ambos. El tiempo de permanencia de los visitantes en el Jardins es, por término medio, superior en más de 30 minutos al del Rio Mar. El gasto medio mensual por visitante y el número medio de visitas al mes, sin embargo, no difiere significativamente.

El tiempo medio de desplazamiento es -aproximadamente- 5 minutos superior cuando se trata de llegar a Jardins que a Rio Mar. Ya indicábamos en la presentación de las características de los centros, que Rio Mar tiene buenos accesos en coche propio o mediante transporte público. No obstante, el número de ciudadanos que se acercan a pie a Jardins es notablemente superior al de Rio Mar, dada su ubicación más céntrica. 
Revista Eletrônica de Ciência Administrativa (RECADM) - ISSN 1677-7387

Faculdade Cenecista de Campo Largo - Coordenação do Curso de Administração v. 2, n. 1, maio/2003 - http://revistas.facecla.com.br/index.php/recadm/

CUADRO 8

Descripción de la muestra

\begin{tabular}{|c|c|c|c|c|}
\hline & & \multicolumn{2}{|c|}{ No (1) } & $\mathrm{Si}(2)$ \\
\hline $\begin{array}{l}\text { Conocen Rio Mar } \\
\text { Conocen Jardins }\end{array}$ & & \multicolumn{2}{|c|}{$\begin{array}{l}0,0 \%(0) \\
0,0 \%(0)\end{array}$} & $\begin{array}{l}100 \%(350) \\
100 \%(350)\end{array}$ \\
\hline $\begin{array}{l}\text { Han visitado Rio I } \\
\text { Han visitado Jardi }\end{array}$ & & \multicolumn{2}{|c|}{$\begin{array}{l}0,0 \%(0) \\
0,0 \%(0)\end{array}$} & $\begin{array}{l}100 \%(350) \\
100 \%(350)\end{array}$ \\
\hline $\begin{array}{l}\text { Han comprado en } \\
\text { Han comprado en }\end{array}$ & & \multicolumn{2}{|c|}{$\begin{array}{l}0,0 \%(0) \\
0,0 \%(0)\end{array}$} & $\begin{array}{l}100 \%(350) \\
100 \%(350)\end{array}$ \\
\hline \multicolumn{4}{|c|}{ Frecuencia de las visitas } & Media (desviación) \\
\hline \multicolumn{4}{|c|}{$\begin{array}{l}\text { Número de veces al mes que se visita Rio Mar } \\
\text { Número de veces al mes que se visita Jardins }\end{array}$} & $\begin{array}{l}6,0(8,2) \\
5,6(7,8)\end{array}$ \\
\hline \multicolumn{4}{|c|}{ Duración de la visita* } & Media (desviación) \\
\hline \multicolumn{4}{|c|}{$\begin{array}{l}\text { Tiempo en minutos de permanencia en Rio Mar } \\
\text { Tiempo en minutos de permanencia en Jardins }\end{array}$} & $\begin{array}{c}89,3(66,5) \\
123,9(85,6)\end{array}$ \\
\hline \multicolumn{4}{|c|}{ Gasto mensual medio } & Media (desviación) \\
\hline \multicolumn{4}{|c|}{$\begin{array}{l}\text { Gasto mensual medio en reales en Rio Mar } \\
\text { Gasto mensual medio en reales en Jardins }\end{array}$} & $\begin{array}{l}62,6(130,6) \\
65,7(97,6)\end{array}$ \\
\hline \multicolumn{4}{|c|}{ Tiempo de desplazamiento* } & Media (desviación) \\
\hline \multicolumn{4}{|c|}{$\begin{array}{l}\text { Tiempo medio en minutos para ir a Rio Mar con el transporte habitual } \\
\text { Tiempo medio en minutos para ir a Jardins con el transporte habitual }\end{array}$} & $\begin{array}{l}15,4(5,4) \\
20,7(5,8)\end{array}$ \\
\hline \multicolumn{5}{|c|}{ Forma de acceso al shopping } \\
\hline & Caminando* & Autobús & Taxi & Vehículo propio \\
\hline $\begin{array}{l}\text { Rio Mar } \\
\text { Jardins }\end{array}$ & $\begin{array}{l}0,3 \%(1) \\
8,3 \%(29)\end{array}$ & $\begin{array}{l}40,6 \%(142) \\
40,3 \%(141)\end{array}$ & $\begin{array}{l}5,4 \%(19) \\
4,9 \%(17)\end{array}$ & $\begin{array}{l}53,7 \%(188) \\
46,6 \%(163)\end{array}$ \\
\hline Conjunto & $4,3 \%(30)$ & $40,4 \%(283)$ & $5,1 \%(36)$ & $50,1 \%(351)$ \\
\hline
\end{tabular}

* Diferencias de medias significativas (prueba T para muestras relacionadas) para un nivel de confianza del $95 \%$.

En el conjunto de los atributos y servicios de cada centro es nítida la supremacía del shopping Jardins, con excepción de los ítemes localización, facilidad de aparcamiento, precios más bajos y tranquilidad en las compras, en los que el shopping Rio Mar es mejor evaluado. La mayor cantidad de tiendas y la presencia de cines en el shopping Jardins es un hecho fundamental en la diferenciación con Rio Mar, que hace que el flujo de visitantes al shopping Jardins sea mayor. Finalmente, en la línea de lo señalado, valga significar que el shopping Jardins es significativamente mejor valorado que el shopping Rio Mar. 
Revista Eletrônica de Ciência Administrativa (RECADM) - ISSN 1677-7387

Faculdade Cenecista de Campo Largo - Coordenação do Curso de Administração v. 2, n. 1, maio/2003 - http://revistas.facecla.com.br/index.php/recadm/

CUADRO 9

Evaluación de los atributos de los shoppings

\begin{tabular}{|c|c|}
\hline Valoración de los atributos de los shoppings & Media (desviación) \\
\hline $\begin{array}{l}\text { Proximidad Rio Mar * } \\
\text { Proximidad Jardins * }\end{array}$ & $\begin{array}{l}3,37(0,66) \\
3,88(0,86) \\
\end{array}$ \\
\hline $\begin{array}{l}\text { Localización Rio Mar * } \\
\text { Localización Jardins * }\end{array}$ & $\begin{array}{l}3,90(0,83) \\
3,75(0,86)\end{array}$ \\
\hline $\begin{array}{l}\text { Facilidad aparcamiento Rio Mar * } \\
\text { Facilidad aparcamiento Jardins * }\end{array}$ & $\begin{array}{l}4,16(0,95) \\
2,33(1,05)\end{array}$ \\
\hline $\begin{array}{l}\text { Precios bajos Rio Mar * } \\
\text { Precio bajos Jardins * }\end{array}$ & $\begin{array}{l}3,29(0,91) \\
3,09(0,99)\end{array}$ \\
\hline $\begin{array}{l}\text { Buena calidad Rio Mar * } \\
\text { Buena calidad Jardins * }\end{array}$ & $\begin{array}{l}3,95(0,34) \\
4,08(0,47)\end{array}$ \\
\hline $\begin{array}{l}\text { Atención al cliente Rio Mar } \\
\text { Atención al cliente Jardins }\end{array}$ & $\begin{array}{l}3,79(0,65) \\
3,78(0,73)\end{array}$ \\
\hline $\begin{array}{l}\text { Limpieza y cuidados Rio Mar* } \\
\text { Limpieza y cuidados Jardins } *\end{array}$ & $\begin{array}{l}3,93(0,39) \\
4,08(0,60)\end{array}$ \\
\hline $\begin{array}{l}\text { Tranquilidad en las compras Rio Mar * } \\
\text { Tranquilidad en las compras Jardins * }\end{array}$ & $\begin{array}{l}3,34(1,04) \\
3,17(0,94)\end{array}$ \\
\hline $\begin{array}{l}\text { Buena reputación Rio Mar* } \\
\text { Buena reputación Jardins * }\end{array}$ & $\begin{array}{l}4,17(0,41) \\
4,52(0,73)\end{array}$ \\
\hline $\begin{array}{l}\text { Variedad de tiendas Rio Mar* } \\
\text { Variedad de tiendas Jardins * }\end{array}$ & $\begin{array}{l}3,23(0,80) \\
3,90(0,82)\end{array}$ \\
\hline $\begin{array}{l}\text { Variedad de servicios Rio Mar } \\
\text { Variedad de servicios Jardins }\end{array}$ & $\begin{array}{l}3,73(0,69) \\
3,78(0,60)\end{array}$ \\
\hline $\begin{array}{l}\text { Entretenimiento Rio Mar * } \\
\text { Entretenimiento Jardins * }\end{array}$ & $\begin{array}{l}3,41(1,00) \\
3,95(0,75)\end{array}$ \\
\hline Valoración global de los shoppings* & Media (desviación) \\
\hline $\begin{array}{c}\text { Rio Mar } \\
\text { Jardins }\end{array}$ & $\begin{array}{l}4,2(0,42) \\
4,5(0,72) \\
\end{array}$ \\
\hline
\end{tabular}

Respecto al tipo de compras y servicios usados en cada uno de los shoppings, excepto para ropa y calzado, donde se encuentra una diferencia significativa a favor de Rio Mar, en el resto de productos y servicios en los que existen diferencias entre los shoppings son favorables a Jardins. 
Revista Eletrônica de Ciência Administrativa (RECADM) - ISSN 1677-7387

Faculdade Cenecista de Campo Largo - Coordenação do Curso de Administração v. 2, n. 1, maio/2003 - http://revistas.facecla.com.br/index.php/recadm/

CUADRO 10

Tipos de compras en los shoppings

\begin{tabular}{|c|c|}
\hline Realización de compras y uso de servicios & Media (desviación) \\
\hline $\begin{array}{l}\text { Alimentación en Rio Mar * } \\
\text { Alimentación en Jardins } *\end{array}$ & $\begin{array}{l}2,94(0,70) \\
3,14(0,59)\end{array}$ \\
\hline $\begin{array}{l}\text { Música y libros en Rio Mar * } \\
\text { Música y libros en Jardins * }\end{array}$ & $\begin{array}{l}2,71(0,73) \\
2,93(0,83)\end{array}$ \\
\hline $\begin{array}{l}\text { Ropa y calzado en Rio Mar* } \\
\text { Ropa y calzado en Jardins * }\end{array}$ & $\begin{array}{l}3,08(0,60) \\
2,86(0,93)\end{array}$ \\
\hline $\begin{array}{l}\text { Electrodomésticos y muebles en Rio Mar * } \\
\text { Electrodomésticos y muebles en Jardins * }\end{array}$ & $\begin{array}{l}2,56(0,78) \\
3,22(0,62)\end{array}$ \\
\hline $\begin{array}{l}\text { Juguetes en Rio Mar * } \\
\text { Juguetes en Jardins * }\end{array}$ & $\begin{array}{l}2,96(0,55) \\
3,18(0,80)\end{array}$ \\
\hline $\begin{array}{l}\text { Cosméticos y perfumería en Rio Mar * } \\
\text { Cosméticos y perfumería en Jardins * }\end{array}$ & $\begin{array}{l}2,94(0,50) \\
3,24(0,61)\end{array}$ \\
\hline $\begin{array}{l}\text { Agencia bancaria en Rio Mar } \\
\text { Agencia bancaria en Jardins }\end{array}$ & $\begin{array}{l}3,06(0,74) \\
3,11(0,55) \\
\end{array}$ \\
\hline $\begin{array}{l}\text { Caja electrónica en Rio Mar } \\
\text { Caja electrónica en Jardins }\end{array}$ & $\begin{array}{l}3,13(0,63) \\
3,07(0,60)\end{array}$ \\
\hline $\begin{array}{l}\text { Peluquería y belleza en Rio Mar * } \\
\text { Peluquería y belleza en Jardins * }\end{array}$ & $\begin{array}{l}2,82(0,96) \\
3,07(0,90)\end{array}$ \\
\hline $\begin{array}{l}\text { Loterías y juegos en Rio Mar } \\
\text { Loterías y juegos en Jardins }\end{array}$ & $\begin{array}{l}3,11(0,60) \\
3,08(0,58)\end{array}$ \\
\hline $\begin{array}{l}\text { Correos en Rio Mar } \\
\text { Correos en Jardins }\end{array}$ & $\begin{array}{l}3,12(0,59) \\
3,14(0,61) \\
\end{array}$ \\
\hline $\begin{array}{l}\text { Restaurantes en Rio Mar } \\
\text { Restaurantes en Jardins }\end{array}$ & $\begin{array}{l}3,21(0,86) \\
3,19(0,74) \\
\end{array}$ \\
\hline $\begin{array}{l}\text { Cine y ocio en Rio Mar * } \\
\text { Cine y ocio en Jardins * }\end{array}$ & $\begin{array}{l}1,87(1,08) \\
4,13(0,69)\end{array}$ \\
\hline
\end{tabular}

En resumen, para cumplir con los objetivos que nos hemos propuesto tenemos información de dos shoppings, conocidos y frecuentados por la población de Aracaju, relativamente próximos, pero con diferentes rasgos o atributos. El Rio Mar se caracteriza por su fácil acceso y aparcamiento, precios bajos y la posibilidad de realizar la compra en un entorno tranquilo. Por su parte, el Jardins, más nuevo y moderno, próximo al centro pero con problemas de acceso y aparcamiento, se caracteriza por el mayor flujo de visitas y tiempo de permanencia, la variedad de tiendas, la buena reputación y el entretenimiento que proporciona.

\section{Resultados}

Los datos presentados en el cuadro 8 revelan el cumplimiento de la primera hipótesis relativa a que los individuos dentro de una determinada zona espacial, ni visitan ni compran en un único centro, sino que tienden a visitar y repartir sus compras entre varios de ellos. Todos los encuestados han visitado y han comprado en los dos centros.

Para el contraste de la segunda hipótesis hemos utilizado el análisis de regresión. Obsérvense en el Cuadro 11 los resultados de los 16 análisis realizados (4 variables de atracción por 2 de distancia y por 2 centros comerciales). En su mayoría, se observa la no existencia de una relación entre la atracción comercial y la distancia. Particularmente cuando la forma de medición es la proximidad al centro, ya que de las 8 regresiones realizadas ninguna se muestra significativa para esta variable independiente. Respecto a la variable tiempo de desplazamiento, se encuentran dos relaciones significativas en el conjunto de las ocho. En definitiva, podemos decir que la variable distancia no se muestra relevante en la atracción ejercida por los centros comerciales. 
Revista Eletrônica de Ciência Administrativa (RECADM) - ISSN 1677-7387

Faculdade Cenecista de Campo Largo - Coordenação do Curso de Administração

v. 2, n. 1, maio/2003 - http://revistas.facecla.com.br/index.php/recadm/

\section{CUADRO 11}

Resultados de los análisis de regresión

\begin{tabular}{|c|c|c|c|c|c|}
\hline $\begin{array}{c}\text { VARIABLE } \\
\text { DEPENDIENTE }\end{array}$ & $\begin{array}{c}\text { VARIABLE } \\
\text { INDEPENDIENTE }\end{array}$ & $\begin{array}{c}\text { CENTRO } \\
\text { COMERCIAL }\end{array}$ & $\begin{array}{c}\text { Beta* } \\
\text { estandarizada }\end{array}$ & $\mathbf{R}^{2}$ & t (Sig.) \\
\hline \multirow{4}{*}{$\begin{array}{c}\text { Frecuencia de las } \\
\text { visitas }\end{array}$} & \multirow{2}{*}{$\begin{array}{c}\text { Tiempo de } \\
\text { desplazamiento }\end{array}$} & Rio Mar & 0,03 & $0,1 \%$ & $0,63(0,53)$ \\
\hline & & Jardins & $-0,01$ & $0,0 \%$ & $-0,17(0,86)$ \\
\hline & \multirow{2}{*}{ Proximidad al centro } & Rio Mar & 0,04 & $0,2 \%$ & $0,78(0,43)$ \\
\hline & & Jardins & $-0,07$ & $0,2 \%$ & $-1,23(0,22)$ \\
\hline \multirow{4}{*}{ Duración de las visitas } & \multirow{2}{*}{$\begin{array}{c}\text { Tiempo de } \\
\text { desplazamiento }\end{array}$} & Rio Mar & 0,11 & $1,2 \%$ & $2,03(0,04)$ \\
\hline & & Jardins & $-0,04$ & $0,2 \%$ & $-0,73(0,47)$ \\
\hline & \multirow{2}{*}{ Proximidad al centro } & Rio Mar & $-0,04$ & $0,2 \%$ & $-0,83(0,41)$ \\
\hline & & Jardins & 0,06 & $0,4 \%$ & $1,29(0,23)$ \\
\hline \multirow{4}{*}{ Gasto mensual } & \multirow{2}{*}{$\begin{array}{c}\text { Tiempo de } \\
\text { desplazamiento }\end{array}$} & Rio Mar & $-0,04$ & $0,1 \%$ & $-0,64(0,52)$ \\
\hline & & Jardins & $-0,04$ & $0,2 \%$ & $-0,79(0,43)$ \\
\hline & \multirow{2}{*}{ Proximidad al centro } & Rio Mar & 0,06 & $0,0 \%$ & $0,09(0,92)$ \\
\hline & & Jardins & 0,03 & $0,1 \%$ & $0,61(0,54)$ \\
\hline \multirow{4}{*}{$\begin{array}{c}\text { Intención } \\
\text { de visita }\end{array}$} & \multirow{2}{*}{$\begin{array}{c}\text { Tiempo de } \\
\text { desplazamiento }\end{array}$} & Rio Mar & 0,05 & $0,0 \%$ & $0,94(0,35)$ \\
\hline & & Jardins & 0,10 & $1,1 \%$ & $1,95(0,05)$ \\
\hline & \multirow{2}{*}{ Proximidad al centro } & Rio Mar & $-0,08$ & $0,7 \%$ & $-1,57(0,11)$ \\
\hline & & Jardins & $-0,02$ & $0,0 \%$ & $-0,34(0,73)$ \\
\hline
\end{tabular}

* Coincide con el coeficiente de correlación entre la variable dependiente y la independiente.

Obviamente, con esto no queda demostrado que la distancia sea irrelevante. Entendemos de dos situaciones donde puede ser un significativo criterio de decisión:

B Cuando la distancia entre los centros es importante.

B Cuando la diferencia de distancia a los centros es pequeña, pero estos son muy similares.

\section{Implicaciones estratégicas y limitaciones}

En las ciudades pequeñas-medianas, los promotores de centros comerciales deben saber que si la distancia media respecto a la población es similar o presenta diferencias menores respecto a otros centros, y sí van a tener un diferente posicionamiento, es pertinente dar entrada a otros criterios de decisión como el precio del suelo, la disponibilidad de espacio para la construcción u otros factores ambientales. Buscar un posicionamiento diferente puede ser una salvaguarda cuando incluso la distancia media al núcleo de población va a ser sensiblemente superior a la media.

El trabajo presenta una serie de limitaciones que, de no darse, nos permitirían mayor firmeza en las afirmaciones.

- Recogida de información en los centros comerciales

Considerar la irrelevancia de las pequeñas diferencias en la distancia cuando los centro son altamente similares Lineas futuras LIMDEP

\section{Bibliografía}

ARnold, S.J.; V. Roth y D. TigerT (1981): "Conditional Logit versus MDA in the Prediction of Store Choice" Advances in Consumer Research, vol. 9, págs. 665-670.

Borgers, A. Y H. Timmermans (1987): "Choice Model Specification, Substitution and Spatial Structure Effects. A Simulation Experiment”. Regional Science and Urban Economics, vol. 17, nº 1, págs. 29-47.

CARLson, H.J. (1991):“The Role of the Shopping Centre in US Retailing”, The International Journal of Retail \& Distribution Management, vol. $19 \mathrm{n}^{\circ}$. 6, pp. 13-20.

DE JUAN VigaRAY, D. Y J. Rivera (1998): "Revisión del constructo atracción comercial: una aproximación teórica”. X Encuentro de Profesores Universitarios de Marketing, 24 al 25 de septiembre, Santander, págs. 345-361. 


\section{Revista Eletrônica de Ciência Administrativa (RECADM) - ISSN 1677-7387 Faculdade Cenecista de Campo Largo - Coordenação do Curso de Administração v. 2, n. 1, maio/2003 - http://revistas.facecla.com.br/index.php/recadm/}

Finn, A. , S. McQuiTTy y J. Rigby (1994): “Residents’ Acceptance and Use of a Mega-multi-mall: West Edmonton Mall Evidence”. International Journal of Research in Marketing, vol. 11, págs. 127-144.

GAuTSCHI, D.A. (1981): “Specification of Patronage Models for Retatil Center Choice”. Journal of Marketing Research, 18, mayo, págs. 162-174.

GENTRY, J.W. y A. BURNS (1977-1978): "How Important are Evaluative Criteria in Shopping Centre Patronage". Journal of Retailing, vol. 53, $\mathrm{n}^{\mathrm{0}} 4$, págs. 73-85.

GonzÁlez BEnito, O. (2000): "Competencia espacial intraurbana de las cadenas líderes de hipermercados en España: modelización explicativa en el marco de la estrategia de localización”. Revista Española de Investigación de Marketing ESIC, vol. $4, \mathrm{n}^{\circ} 1$, págs. 113-141.

HAUSER, J.R. y F.S. KoPPELMAN (1979): “Alternative Perceptual Mapping Techniques: Relative Accuracy and Uselfuness". Journal of Marketing Research, 16, págs. 495-506.

Howell, R. y J.D. Rogers (1980): "Research into Shopping Mall Choice Behavior". En Advances in Consumer Research, K. Monroe (editor), vol. 8, Association for Consumer Research, Ed. Ann Arbor, págs. 671-681.

KaYLing, S.O. (1973): “In Depth Analysis Necessary for Shopping Center Game”, Shopping Centre World, pp. 43-48.

ManZo, J.M. y W. Kunto (1975): Marketing para ejecutivos. 6. ed. Rio de Janeiro, Zahar, 1975, p. 300.

MÁs Ruiz, F. (1996A): “Atracción de Centros Comercial en el contexto de la elección discreta individual”. Instituto Valenciano de Investigaciones Económicas, WP-EP, Valencia, págs. 96-117.

MÁs RuzZ, F. (1996B): "La imagen de los centros comerciales surburbanos en el modelo de Huff: una aplicación empírica". X Congreso Nacional de AEDEM, págs. 165-177, Granada.

MÁs RuIz, F. (1999): "Image of Suburban Shopping Malls and Two-Stage Versus Uniequation Modelling of the Retail Trade Atracction”. European Journal of Marketing, vol. 33, nº 5/6, págs. 512-530.

MedinA, O. (1998): "El modelo de gravitación comercial en España: Un análisis de los flujos de gastos comerciales por categoría de productos". Revista Española de Investigación de Marketing ESIC, vol.2 , nº 1, págs. 113-134.

MCGoldRICK, P. (1992): "El desarrollo en Europa de centros de compra a gran escala fuera de las ciudades". En La distribución en el 2000, Colección de Estudios Comerciales, Ed. Generalitat Valenciana, Valencia.

Nevin, J.R. Y M.J. Houston (1980): "Image as a Component of Attraction to Intraurban Shopping Areas". Journal of Retailing, vol. $56, \mathrm{n}^{\circ} 1$, págs. 77-93.

Rust, R.T. Y N. Donthu (1995): “Capturing Geographically Localized Misspecification Error in Retail Store Choice Models. Journal of Marketing Research, vol. 32, febrero, págs. 103-110.

Spiggle, S. y M.A. Sewall (1987): “A Choice Sets Model of Retail Selection”, Journal of Marketing, vol. 51, abril, págs. $97-111$.

Stolman, J.J., J.W. Gentry y K.A. Anglin (1991): "Shopping Choices: The Case of Mall Choice”, Advances in Consumer Research, vol. 18, págs. 434-440.

SuÁREZ VAZQueZ, A.M., (1999): "Atracción de centros comerciales: modelización de estructuras de decisión alternativas", Tesis doctoral no publicada, Universidad de Oviedo, Departamento de Administración de Empresas y Contabilidad.

WeE, C.H. (1986): "Shopping Area Image: Its Factor Analytic Structure and Relationships with Shopping Trips and Expenditure Behavior”. En Advances in Consumer Research, R.J. Lutz (editor), Association for Consumer Research, págs. 49-52.

WeE, C.H. y M.R. Pearce (1985): "Patronage Behavior Toward Shopping Areas: A Proposed Model Based on Huff's Model of Retail Gravitation". En E.C. Hirschman y M.B. Holbrook (editores), Advances in Consumer Research, vol. 12, Association for Consumer Research, págs. 592-597.

Weisbrod, G.E., R.J. Parcells y C. Kern (1984): “A Disaggregate Model for Predicting Shopping Area Market Attraction”. Journal of Retailing, vol. 60, $\mathrm{n}^{\circ}$ 1, págs. 56-83. 